\title{
Análisis estructural para la determinación de variables clave en el sistema de educación ambiental (EA) de la niñez temprana peruana
}

\author{
Structural analysis to determine the key variables of the Peruvian early childhood \\ environmental education (EE) system
}

\section{Análise estrutural para determinar as principais variáveis do sistema peruano de educação ambiental (EA) da primeira infância}

\author{
Almendra Farah-Sprinckmoller ${ }^{1}$ \\ Alessandra Navarro ${ }^{2}$ (D) \\ Marcos Fernando Ruiz-Ruiz ${ }^{3}$
}

Recibido: mayo de 2020

Aceptado: octubre de 2020

Para citar este artículo: Farah-Sprinckmoller, A., Navarro, A., Ruiz-Ruiz, M. F. (2021). Análisis estructural para la determinación de variables clave en el sistema de educación ambiental (EA) de la niñez temprana peruana. Revista Científica, 40(1), 30-44. https://doi.org/10.14483/23448350.16288

\section{Resumen}

El objetivo de este estudio fue determinar las variables claves que caracterizan el sistema de educación ambiental (EA) peruano necesario para su niñez temprana, así como qué actores lo conducen hacia el futuro. A partir de un diseño exploratorio y descriptivo, y con la ayuda de un grupo de cinco educadores expertos en la temática y de reconocida trayectoria local, se efectuó un análisis estructural (propio de la prospectiva estratégica francesa) orientado a la identificación de las variables clave del sistema. Los resultados arrojaron como aspectos más sensibles de la EA de los niños peruanos, al aprendizaje desde la realidad local hacia lo global, a la promoción de acciones sostenibles y a las acciones solidarias y de emprendimiento docente en las comunidades. El actor más influyente para el sistema termina siendo el propio Ministerio de Educación del Perú (Minedu), pues es responsable de conducir la EA de la niñez peruana hacia mejores estándares de calidad en el futuro.

Palabras clave: educación ambiental, análisis estructural, niños de preescolar, planeamiento educativo, Perú.

\section{Abstract}

The objective of this study was to determine the key variables that characterize the Peruvian Environmental Education (EE) system necessary for early childhood, as well as which actors lead it into the future. Based on an exploratory and descriptive design, and with the help of a group of five expert educators on the subject and with a recognized local background, a structural analysis, typical of French

1. Instituto Tecnológico y de Estudios Superiores de Monterrey: Monterrey, Nuevo León, México. A01313024@itesm.mx

2. Instituto Tecnológico y de Estudios Superiores de Monterrey: Monterrey, Nuevo León, México. ruthrdz@tec.mx

3. Instituto Tecnológico y de Estudios Superiores de Monterrey: Monterrey, Nuevo León, México. ruthrdz@tec.mx 
strategic prospective, was carried out, aimed at determining the key variables of the system. The results showed that the most sensitive aspects of the EE of Peruvian children are learning from the local reality to the global, the promotion of sustainable actions, and solidarity actions and teacher entrepreneurship in the communities. The most influential actor for the system ends up being the Ministry of Education of Peru (Minedu) itself as the agent responsible for driving the EE of Peruvian children towards better quality standards in the future.

Keywords: environmental education, structural analysis, preschool children, educational planning, Peru.

\section{Resumo}

O objetivo deste estudo foi determinar as variáveis-chave que caracterizam o sistema de Educação Ambiental (EA) peruano necessário para a primeira infância, bem como quais atores o conduzem para o futuro. Com base em um projeto exploratório e descritivo, e com a ajuda de um grupo de cinco educadores especializados sobre o assunto e com um histórico local reconhecido, foi realizada uma análise estrutural, típica da perspectiva estratégica francesa, com o objetivo de determinar as principais variáveis do sistema. Os resultados mostraram que os aspectos mais sensíveis da EA das crianças peruanas são aprender da realidade local ao global, promover ações sustentáveis e as ações solidárias e o empreendedorismo de professores nas comunidades. $\mathrm{O}$ ator mais influente do sistema acaba sendo o próprio Ministério da Educação do Peru (Minedu) como o agente responsável por conduzir a EA das crianças peruanas em direção a melhores padrões de qualidade no futuro.

Palavras-chaves: educação ambiental, análise estrutural, pré-escolares, planejamento educacional, Peru.

\section{Introducción}

En la actualidad el mundo enfrenta las consecuencias del cambio climático y el calentamiento global (Asian Development Bank [ADB], 2015). El Intergovernmental Panel on Climate Change (IPCC, 2013) y el ADB (2015) mencionan que el desarrollo industrial de la humanidad es una de las causas más importantes del aumento en las concentraciones de gases de efecto invernadero (GEI) desde mediados del siglo XIX. Estos cambios se pueden evidenciar en el calentamiento de océanos, el deshielo de glaciales, el aumento del nivel del mar, el aumento de concentraciones de los GEI, la persistencia de temperaturas extremas, inundaciones, sequías y aumento en la temperatura promedio global cada año desde 1850 (IPCC, 2013; U.S. Global Change Research Program, 2018). La población más afectada por las emergencias mundiales de hoy está conformada por niños entre 0 y 8 años que son vulnerables y que, además, dependen física y psicológicamente de las decisiones de los gobiernos (Adamson, 2008; Fondo de las Naciones Unidas para la Infancia [Unicef], 2010). Frente a estas alteraciones ambientales, la sociedad ha experimentado cambios demográficos relacionados a la creciente urbanización, lo que ha provocado pérdida de zonas naturales (Marcén, 2014). En el Perú, el 79,3 \% de las personas viven en centros poblados urbanos y el 20,7 \% en centros poblados rurales (Instituto Nacional de Estadística e Informática, 2017), lo cual acarrea que la población peruana transite en ciudades dominadas por cemento, delimitadas por paredes y con cada vez menos espacios verdes (Tonucci, 2020).

Según la Organización Mundial de la Salud, una ciudad sostenible debe contar con $9 \mathrm{~m}^{2}$ de espacio verde por habitante (Sistema Nacional de Información Ambiental, 2020). Sin embargo, en el Perú esta cifra apenas supera los $3 \mathrm{~m}^{2}$ por habitante en ciudades como Lima, Arequipa y Tacna (Torrico, 2020). En este sentido, tanto adultos como niños se ven afectados debido a que pasan la mayor parte del tiempo en espacios interiores, perdiendo así el contacto con el mundo exterior y los aprendizajes que pueden vivenciar (Tonucci, 2019); asimismo, la revolución tecnológica brinda nuevas experiencias sin necesidad de movilidad ni contacto social (Waller et al., 2010). Louv (2008) atribuye el término trastorno por déficit de la naturaleza a los niños que no tienen suficientes experiencias de juego libre ni un vínculo con la naturaleza. 
Debido tanto a esta situación, como a los problemas de conservación y explotación del medioambiente, debe abordarse la preservación y respeto desde un punto de vista educativo, antes que formulando leyes y reglamentos (Morachimo y Piscoya, 2004). Teniendo en cuenta esta preocupación por el medioambiente, desde el campo de la pedagogía surge la propuesta de educación ambiental (EA). Leff (1998) menciona que este tipo se origina de la necesidad por orientar la educación en el contexto social, así como en la realidad ecológica y cultural en la que se sitúan los sujetos y actores del proceso educativo. Según Morachimo y Piscoya (2004), la EA tiene que "ofrecer" antes que desarrollar capacidades en las personas, para asegurarse un ingreso "ecológicamente viable" o la "capacidad para preocuparse por el ambiente" o la "capacidad para protestar". En esta línea, la EA ha sufrido un cambio progresivo de paradigmas.

A fines del siglo XIX nació el modelo de EA Ilamado "naturalista", el cual busca el desarrollo a partir del crecimiento económico (Morachimo y Piscoya, 2004). Por un lado, en la escuela se realizaron campañas con el objetivo de desarrollar una actitud de conservación del ambiente mediante la transmisión de contenidos sobre los espacios naturales y la extinción de animales; mientras que las políticas nacionales se orientaron a conservar espacios y convertirlos en reservas naturales (International Union for Conservation of Nature, 1972). En la década de 1970 la EA modificó su acción y objetivos. Desde la escuela surgió un segundo enfoque denominado "ecologista", en el que se propiciaba el desarrollo de un conjunto de capacidades y de conocimientos sobre el medioambiente, los ecosistemas y los recursos naturales a través del curso o asignatura de Ecología. El objetivo era crear una conciencia o moral ambiental, la cual no solo se promueve al ofrecer información sobre problemas ambientales, sino también a través del vínculo entre personas y ambiente (Morachimo y Piscoya, 2004; Wensing y Torre, 2009).

El transmitir conocimientos y desarrollar habilidades y capacidades no era suficiente para el cuidado y la conservación del medioambiente. Por esto es por lo que surge el enfoque denominado "ambientalista", el cual exige dejar el modelo antropocéntrico y preocuparse no solo por el bienestar de los humanos, sino del cuidado de las posibilidades de mantener la diversidad biológica y cultural en el planeta (Morachimo y Piscoya, 2004; Castillo et al., 2017). La relación que establecen los individuos con su comunidad crea un sentido de pertenencia y configura una forma de ver y situarse en el mundo (Novo, 2006). A partir de ello nace la idea de ciudadanía planetaria como concepto ecocéntrico; es decir, la concepción del ser humano como un ser ecodependiente y que está incluido en el entorno (Novo y Murga 2010). Este último surge por el enfoque de desarrollo sostenible, que es un nuevo paradigma que orienta las prácticas y decisiones en cuanto a valores: gestión, criterios económicos, sociales y medioambientales (Novo, 2009). Desde esta perspectiva, la sostenibilidad es una estrategia de desarrollo conducente a satisfacer las necesidades del presente sin comprometer la capacidad de las generaciones futuras (World Commission on Environment and Development [WCED], 1987; Sen, 2013).

Tomando en cuenta que la EA es parte de los objetivos de desarrollo sostenible, se entiende que esta es una acción educativa que fomenta la toma de conciencia acerca del entorno en el que vivimos (ONU, 2019). Este saber ambiental requiere el reconocer valores y conceptos para que las personas puedan desarrollar habilidades y actitudes necesarias y así comprender, apreciar y tomar decisiones acerca de la interrelación del hombre con la cultura y su entorno biofísico (Díaz-Flores, 2008; Tanner, 2010; Mishra, 2016). Su objetivo formativo es desarrollar la conciencia ambiental acerca de problemas ambientales, acciones, hábitos, actitudes, comportamientos y valores proambientales, así como soluciones que puedan adaptarse y contribuir a la sociedad (International Union for Conservation of Nature, 1972; Mishra, 2016; Flórez-Yepes et al., 2018). En efecto, la EA busca dar respuesta a los desafíos que se presentan en 
nuestra época de manera crítica y ética (Gouvêa, 2006). La EA y la formación continua sobre la problemática ambiental aseguran el éxito a largo plazo en cuanto a políticas ambientales, porque de esta manera una sociedad consciente y responsable asegurará la creación de estos programas y su continuidad (Greenall y Womersley, 1977). Sobre este saber ambiental indican diversos autores que debería llevarse desde la niñez temprana hasta la adultez como un aprendizaje permanente.

Por otro lado, la educación inicial es pensada como el periodo crucial para niños y niñas debido al rápido desarrollo a nivel cognitivo, físico y social (ONU, 2006). Asimismo, es un periodo en el que es posible la sensibilización ante problemas ambientales y en el que se forjan hábitos y comportamientos (Giollito, 1984). A partir de esta sensibilización, se construyen los aspectos fundamentales de su identidad ambiental y se establecen fundamentos, valores, actitudes y la moral requerida para un desarrollo sostenible ambiental y para saber cómo enfrentar la problemática ambiental (Gregers, 2013; Green, 2015; Eunhye et al., 2016). Según Davis (1998) y Russo (2001), es en la educación de la niñez temprana donde la EA funciona como un "aliado natural"; es decir, al trabajar de manera interdisciplinar y holística, con un fuerte vínculo entre el juego al aire libre y el aprendizaje de los niños. Estas experiencias directas con la naturaleza y la manipulación de materiales naturales ofrecen oportunidades para apreciar nuestro entorno y vincularnos con este, lo que permite generar una base sólida para la construcción de relaciones sostenibles con la naturaleza y la sociedad. Por esto, lo que se necesita es una escuela con un enfoque de desarrollo holístico, donde el juego y exploración en la naturaleza permitan a los niños aprender y lograr las competencias esperadas; en la cual los sentidos formen parte y permitan descubrir la sabiduría del entorno y el sentido global de pertenencia al mundo (Bruchner, 2012; Carson, 1998; Freire, 2011; Fjørtoft, 2001).

Para fomentar dicha conciencia ambiental en futuras generaciones es importante reconocer el papel que cumplen los docentes en los procesos de enseñanza-aprendizaje. Se afirma que la calidad del sistema educativo de un país está condicionada por la calidad de sus docentes. Es decir, docentes competentes repercutirán de forma directa en los procesos de aprendizaje de sus estudiantes, lo cual se ve reflejado también en la calidad educativa y en los procesos de aprendizaje (Bruns y Luque, 2014; Tan, 2015; Mizala y Schneider, 2019). Por esta razón, se considera de suma importancia la formación y capacitación docente para el abordaje de temas de sostenibilidad (Organización de las Naciones Unidas para la Educación, la Ciencia y la Cultura [Unesco], 2005; McKeown, 2007). Según Bowers (2002), todo humano tiene la obligación moral de actuar en favor de lo natural; y en especial, los docentes deben realizar acciones pedagógicas morales con la madre Tierra que involucren cambios de consumo en el ámbito personal y colectivo, ya que las acciones enseñan más que la teoría. Existen formaciones profesionales para docentes que quieren contribuir a un desarrollo sostenible y armónico. En estas formaciones se promueve: propiciar un ambiente saludable, fomentar las prácticas ambientalmente responsables para la mejora de la calidad ambiental, conectar las prácticas ecológicas con el currículo y las actividades del salón, desarrollar el pensamiento crítico que permita pensar y actuar tanto local como globalmente, trabajar con los conceptos pedagógicos, métodos y técnicas apropiadas para la EA; y aprender a "ambientalizar" los currículos de las diferentes asignaturas (Fundación Universitaria Iberoamericana, 2020; United States Green Building Council [USGBC], 2018).

El énfasis en la EA pretende introducir en el acto educativo los problemas de la sociedad tanto local como globalmente. No solo se dedica a desarrollar capacidades o destrezas individuales para el futuro de las personas en el campo profesional, sino que pretende desarrollar personas críticas que respondan a las demandas actuales y del futuro. Esto supone pensar el desarrollo en términos de sostenibilidad EA como un asunto ético y político (Novo, 2009). 


\section{Revisión de la literatura}

La educación temprana de calidad generaría un impacto significativo en el desarrollo infantil a corto y largo plazo (Clarke-Stewart y Allhusen, 2005; NICHD Early Child Care Research Network, 2005). Para la enseñanza de la EA y sostenibilidad en la niñez temprana diversos autores proponen un enfoque holístico, es decir, un enfoque abierto, estratégico, sostenible e integrado, el cual tiene en cuenta el desarrollo de la EA como condición necesaria para el cuidado y desarrollo de la especie humana (Fedosejeva et al., 2018).

Según Morachimo y Piscoya (2004), el proceso de toma de conciencia y vínculo con el medioambiente va a la par con el desarrollo moral y cognitivo de la persona. Asimismo, pasa por distintas fases: sensibilización, conocimiento, interacción y acción voluntaria. Para realizar una acción voluntaria se requiere de una motivación intrínseca e implica que la persona haya pasado por las fases anteriores y experimentado diversas situaciones que permitan no solo vincularse, sino desarrollar capacidades que puedan también favorecer el medio donde vive. Es decir, desarrollar un compromiso social y político hacia el medio en el que se vive y convive.

Desde Rousseau se hace referencia a una EA, pues hablaba de la naturaleza como la única y verdadera maestra del alumnado, fuente de sabiduría y la cual debemos de cuidar para salvarnos a nosotros mismos (Montero, 2009). Los niños y niñas, en sus primeros años, se encuentran en las etapas de desarrollo sensorio motriz y preoperatorio según Piaget, en el cual exploran, piensan, sienten, actúan y se involucran directamente con el entorno en el que se movilizan (Green, 2015). Torquati et al. (2013) y Eunhye et al. (2016) afirman que experiencias de aprendizaje desarrolladas en la naturaleza permite a niños y niñas crear un vínculo con esta, lo que sería el inicio de la EA.

El desarrollo de las experiencias de vínculo con la naturaleza muestra que niños y niñas pequeños han logrado un aprendizaje significativo sobre la tierra y conceptos básicos sobre temas ambientales, así como la responsabilidad que implica la sostenibilidad (Engdahl, 2015). Barratt-Hacking et al. (2013) y Green (2015) mencionan que, para lograr aprendizajes significativos, en lo que respecta a la EA, idealmente se debe involucrar a niños y niñas en todos los aspectos posibles del proceso de aprendizaje. Es decir, deberían ser parte del proceso de exploración, recolección de información, interpretación, análisis y presentación de resultados; experiencias que responden al nivel de desarrollo en que se encuentren dichos estudiantes. También proponen el uso de otros lenguajes artísticos como medio de comunicación para sus ideas y sentimientos. Esto se puede producir a través de la pintura, construcción, fotografía y el juego de roles, las cuales son actividades que permiten observar los aprendizajes desarrollados sobre todo en la niñez temprana (Green, 2015). El involucrar a los estudiantes en el proceso de aprendizaje es clave para formar personas más competentes, responsables y confiadas en el cambio (Lowe et al., 2016).

Finalmente, otras herramientas para la EA en la niñez temprana son el juego, la exploración del entorno y la aproximación a la naturaleza de una manera científica; es decir, experiencias que le ofrezcan el entendimiento de fenómenos naturales y crear contextos de aprendizaje en los que se simulan diversos ecosistemas. En estas experiencias de aprendizaje los niños tienen la oportunidad de tomar decisiones libremente $y$, a su vez, se necesitan insumos físicos, lúdicos y cognitivos para captar su atención y mantener su interés sobre la estrategia pedagógica (Galeano et al., 2018).

\section{La EA en el caso peruano}

Para el caso peruano, en la década de 1980 y a partir de diversas experiencias, se creó el comité técnico permanente de EA y un programa de educación forestal. A final de esta década se llevó a consolidó el programa de educación ecológica para escuelas rurales y el proyecto Globe 
del Ministerio de Educación del Perú (Minedu, 2019a). En la década de 1990 se consolidó la política de EA y se puso en funcionamiento en todos los niveles educativos. Asimismo, se consolidó el Código Medioambiental y de Recursos Naturales. Estos funcionan como marco jurídico para las implementaciones políticas en las diversas regiones del país (Minedu, 2019b).

Desde año 2002 se han venido realizando esfuerzos de mejora en cuanto a EA, como el Convenio Marco Educacional Ambiental, del que surge la idea de una Política Nacional de Educación Ambiental (PNEA), la cual establece objetivos, lineamientos de política y resultados esperados en la formación y fortalecimiento de una ciudadanía responsable con el ambiente y su entorno, en el marco del desarrollo sostenible nacional (Minedu, 2019c). En el 2003 se expidió la Ley General de EA, la cual menciona a la conciencia ambiental como principio de la educación. En el 2005 se creó la Ley General del Ambiente, en la que se trabajan los lineamientos orientadores de la PNEA. Del 2006 al 2008 se elaboró la PNEA y en el 2011 se establecieron metas para que en el 2012 fuese aprobada. Del 2013 al 2015 el Minedu y el Ministerio del Ambiente (Minam) coordinaron la implementación de la PNEA.

En los mismos años se creó un instrumento de gestión pública el cual es el Plan Nacional de Educación Ambiental 2017-2022 (Planea) (Minam y Minedu, 2016). Este documento se aprobó en 2016 y toma como punto de partida el reconocimiento del ambiente para la participación de la sociedad, con un alto grado de conciencia y cultura ambiental en procesos de gestión ambiental. En del sector educativo el Planea es una herramienta para que la población conozca, comprenda, reflexione y participe proactivamente en las acciones relacionadas a la gestión de emisiones de GEI y la adaptación de cara al cambio climático (Minam y Minedu, 2016).

Por otro lado, el Minedu propone que el trabajo de la EA sea desde el enfoque ambiental mencionado el Currículo Nacional Peruano de Educación
Básica 2016. Este enfoque propone que toda persona sea formada en conciencia crítica y colectiva acerca de la problemática ambiental y desarrollo sostenible local y globalmente. Implica, además, desarrollar los temas de biodiversidad, ecosistemas, producción, consumo responsable, gestión y manejo de residuos, adaptación y gestión ante el cambio climático y desastres naturales y estilo de vida sostenible (Minedu, 2016). Esto mantiene correspondencia con la ya mencionada línea del enfoque ambientalista. Por último, el Proyecto Educativo Nacional Peruano (PEN) para el año 2036 enfatiza que:

Todas las personas en el Perú aprendemos, nos desarrollamos y prosperamos a lo largo de nuestras vidas, ejerciendo responsablemente nuestra libertad para construir proyectos personales y colectivos, conviviendo y dialogando intergeneracional e interculturalmente, en una sociedad democrática, equitativa, igualitaria e inclusiva, que respeta y valora la diversidad en todas sus expresiones y asegura la sostenibilidad ambiental. (Consejo Nacional de Educación [CNE], 2020, p. 24)

Esta visión plantea una ciudadanía inclusiva y equitativa que busca bienestar socioemocional, productividad, prosperidad, investigación y sostenibilidad (CNE, 2020). El proyecto educativo comparte la visión de la agenda 2030 para el desarrollo sostenible, la cual contiene 17 objetivos que, principalmente desde el año 2020 al año 2030, buscan actuar frente a la creciente pobreza, empoderar a las mujeres y a las niñas; así como afrontar la emergencia climática (ONU, 2019, 2020; CNE, 2020).

\section{Metodología}

El método empleado en esta investigación recoge las bases de la prospectiva estratégica de escuela francesa (De Jouvenel, 1964; Godet, 2007). Esta permite determinar los posibles escenarios futuros de un sistema a fin de establecer las mejores 
estrategias orientadas a alcanzar el más conveniente de ellos (Munck y McConnell, 2009). La prospectiva tiene en cuenta la construcción social del futuro y el trabajo de un colectivo humano desde el presente para la búsqueda de alternativas de solución para la construcción del futuro elegido (Godet, 2007; Ruiz-Ruiz, 2017). El diseño Ilevado tiene un carácter exploratorio y descriptivo; teniendo como objetivo analizar un sistema para describirlo a fin de determinar aquellas variables que lo componen y que lo determinan para el futuro. Una técnica útil para este proceso es el análisis estructural. La construcción social del futuro antes mencionada es guiada por el llamado "gen voluntarista"; es decir, la voluntad es la que mueve a los seres humanos en el proceso de construir tal futuro deseado (De Jouvenel, 1964; Munck y McConnell, 2009).

Se realizó una convocatoria a cinco expertos y conocedores de la realidad de la niñez temprana peruana en EA, tanto del sector público como privado. La selección de estos permitió, adicionalmente, la validación continua del proceso seguido, ya que su participación fue activa a través de consultas permanentes. La tabla 1 presenta el perfil de los expertos antes mencionados.

Tabla 1. Expertos convocados (perfiles).

\begin{tabular}{cl}
\hline Código & \multicolumn{1}{c}{ Perfil general } \\
E1 & $\begin{array}{l}\text { Educador del nivel secundaria y experto en } \\
\text { formación y educación de Ciencias Sociales y } \\
\text { Desarrollo Sostenible. } \\
\\
\text { Director ejecutivo de la Asociación para la } \\
\text { Niñez y su Ambiente en el Perú (Ania) y experto } \\
\text { en la gestión ambiental y el papel del niño en el } \\
\text { desarrollo sostenible. }\end{array}$ \\
& $\begin{array}{l}\text { Experto en EA de la Dirección de Educación } \\
\text { E3 Ciudadanía Ambiental del Ministerio del }\end{array}$ \\
& $\begin{array}{l}\text { Ambiente del Perú (Minam). } \\
\text { Cofundador y director de mercadeo y relaciones } \\
\text { públicas de la ONG EcoSwell en Perú. Experto } \\
\text { en temas de desarrollo ambiental y educación. }\end{array}$ \\
& $\begin{array}{l}\text { Educadora con amplia experiencia de } \\
\text { trabajo con la niñez temprana peruana. Con } \\
\text { especialidad en EA. }\end{array}$ \\
\hline
\end{tabular}

Fuente: elaboración propia.

\section{Procedimiento}

A partir de la revisión de literatura y la definición del sistema materia de investigación, se realizó una matriz con las dimensiones y variables constituyentes de la EA para la niñez temprana peruana (tabla 2). La revisión de literatura se desarrolló principalmente en bases de datos como Scopus y Google Scholar, así como en repositorios digitales de tesis de diferentes universidades (entre ellas, la Universidad Complutense de Madrid y la Universidad del Sur de California), revistas especializadas (como el Environmental Research Journal); así como en documentos y páginas web oficiales del Ministerio de Educación del Perú (Minedu). Cada dimensión fue desagregada en un grupo de variables. Se identificaron cuatro dimensiones: las competencias en EA del docente de niñez temprana, la metodología de enseñanza para la EA en la niñez temprana, las competencias para la EA y los recursos para la enseñanza de la EA. Asimismo, para la mejor comprensión de las mencionadas variables, se elaboró un breve alcance de cada una de ellas.

El sistema, junto a sus dimensiones y variables constitutivas, fue validado y ajustado en rondas sucesivas por los expertos convocados. Durante la validación se efectuaron modificaciones y se agregaron nuevas variables. Luego de este proceso se coordinó una última entrevista con los cinco participantes a fin de entrevistarlos sobre el nivel de motricidad y dependencia de todas las variables del sistema y, de esta manera, identificar aquellas variables claves para la evolución de este. Para la entrevista se empleó como herramienta una matriz de motricidad y dependencia, propia de la técnica del análisis estructural (Godet, 2007). El análisis de motricidad y dependencia es una herramienta prospectiva que utiliza el software de Matriz de Impactos Cruzados (MICMAC) y que permite el mapeo de un sistema a partir de la reflexión colectiva realizada con los expertos. Tiene como objetivo reconocer las variables más dependientes y motrices según un puntaje cruzado asignado por los expertos (La prospective, octubre de 2019). 
Tabla 2. Educación Ambiental (EA) para la niñez temprana (dimensiones y variables)

\section{Dimensión: competencias en EA del docente de niñez temprana \\ Variable \\ Alcances}

Nivel de formación ambiental de los docentes de niñez temprana.

Acciones sostenibles en su cotidianidad.

Acciones solidarias y de emprendimiento en la comunidad para el desarrollo sostenible.
Formación profesional en educación con especialización en niñez temprana y en EA.

Incorporación de acciones sostenibles relacionadas con la gestión de recursos (residuos sólidos, uso ecoeficiente de agua y energía, consumo sostenible y valoración de la biodiversidad).

Interacción con la comunidad para contribuir al desarrollo sostenible (fomenta alianzas, acuerdos, redes).

\section{Dimensión: metodologías de enseñanza-aprendizaje para la EA en la niñez temprana \\ Variable \\ Alcances}

Promoción de acciones sostenibles (dentro y fuera del aula).

Promoción de estrategias didácticas para la enseñanza de la EA.

Propiciar experiencias que permitan el aprendizaje significativo.

Aprendizaje a partir de la realidad local hacia la global.
Promoción de acciones sostenibles en la institución educativa (gestión de residuos sólidos, uso ecoeficiente del agua y energía, consumo sostenible y reconocimiento de la biodiversidad).

Uso de estrategias didácticas para el desarrollo de la EA (interacción con la naturaleza, participación activa, cooperación, colaboración, aprendizaje basado en proyectos y problemas, etc.).

Promoción de construcción de saberes y habilidades a partir de preconceptos del niño y niña.

El proceso de enseñanza aprendizaje toma el contexto para atender problemáticas locales y globales.

\section{Variable}

\section{Dimensión: competencias del niño o niña para la EA}

Ciudadanía planetaria.

Construcción de la identidad.

Convivencia y participación democrática en la búsqueda del bien común.

Indagación mediante métodos científicos para la construcción de sus conocimientos

\section{Alcances}

Dar una visión de polis mundial desde la escuela donde todos se consideren parte de una misma región y con un mismo objetivo: el de participar de activamente en las decisiones que comprometan al planeta.

Construcción de su identidad al tomar conciencia de los aspectos que lo hacen único (características físicas, cualidades, intereses, preferencias); sintiéndose miembro de su comunidad y participando de manera autónoma en las decisiones considerando a los otros.

Interacción respetuosa con sus compañeros e interés por conocer más sobre las costumbres y características de todos aquellos dentro de su entorno. Participación y proposición de acuerdos y normas de convivencia orientadas al bien común.

Exploración de objetos, del espacio y de hechos que acontecen en su entorno. Elaboración de preguntas sostenidas en su curiosidad.

\section{Dimensión: recursos para la enseñanza de la EA}

\section{Variable}

Inversión por parte del Estado o colegio para la enseñanza de la EA.

Existencia de recursos y materiales para la enseñanza de la EA.

\section{Alcances}

Inversión económica y asignación de partidas presupuestales específicas por parte del Estado o colegio.

Disposición oportuna y uso pertinente de materiales y recursos para la enseñanza de la EA.

Fuente: elaboración propia. 


\section{Resultados}

El procesamiento de datos con el Micmac constó de tres etapas previas a la obtención de los resultados: el ingreso de las 13 variables identificadas para el sistema, la evaluación por parte de los expertos del nivel de impacto y dependencia entre dichas variables y la identificación de las variables clave para el sistema (las más motrices y dependientes). La relación de impacto entre variables se evaluó en una escala de cero (0) a tres (3), en la que cero (0) significó que impacto nulo, uno (1) bajo impacto, dos (2) impacto medio y tres (3) alto impacto. En la última fase se jerarquizaron las variables para identificar el papel que juegan dentro del sistema. Estas pueden tener relación directa o indirecta. A partir de ello, se llegó a la gráfica de motricidad y dependencia mostrado en la figura 1.

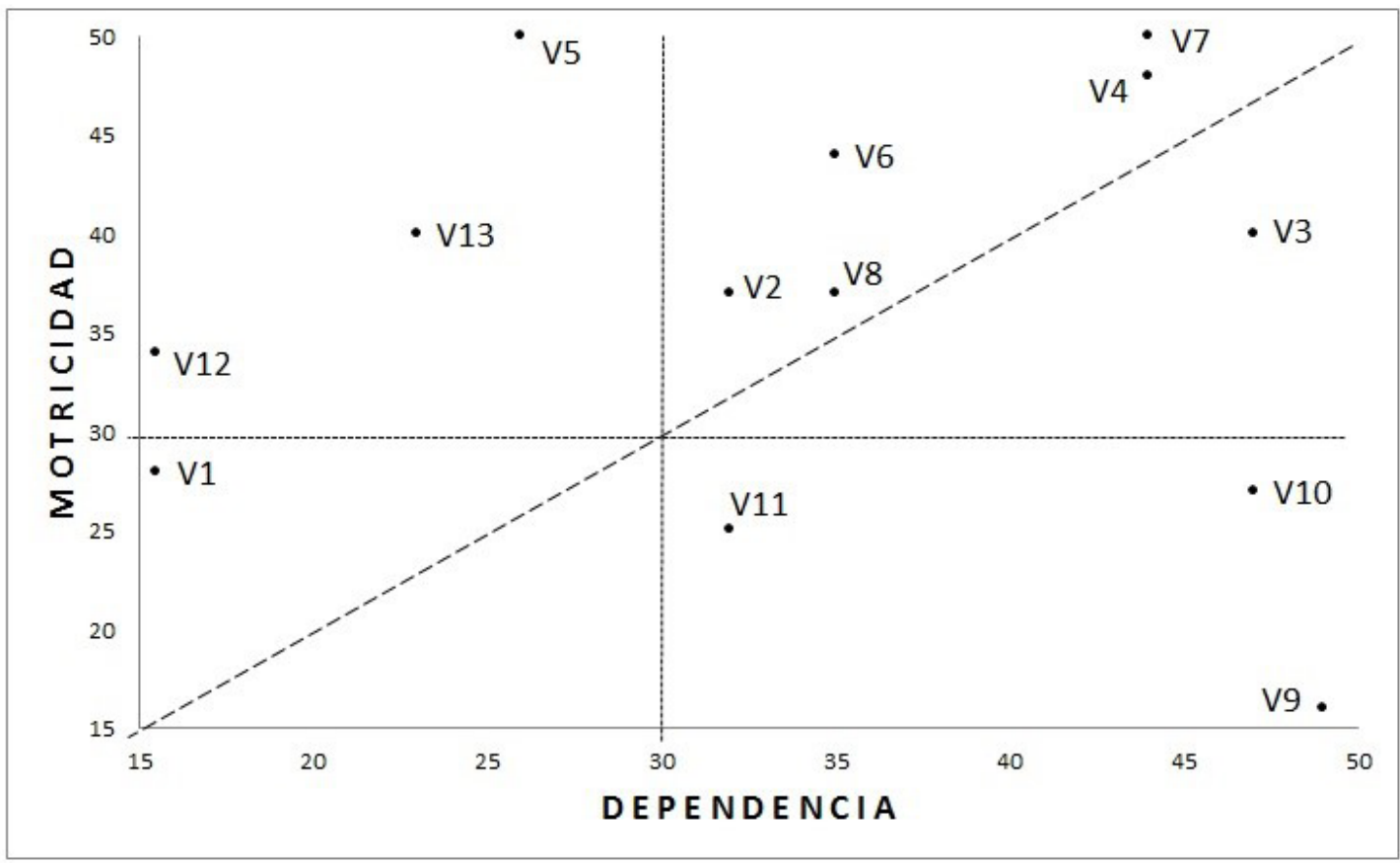

V1: Nivel de formación ambiental de los docentes de niñez temprana.

V2: Acciones sostenibles en su cotidianidad.

V3: Acciones solidarias y de emprendimiento en la comunidad para el desarrollo sostenible.

V4: Promoción de acciones sostenibles (dentro y fuera del aula).

V5: Promoción de estrategias didácticas para la enseñanza de la EA.

V6: Propiciar experiencias que permitan el aprendizaje significativo.
V7: Aprendizaje a partir de la realidad local hacia lo global.

V8: Ciudadanía planetaria.

v9: Construcción de la identidad.

V10: Convivencia y participación democrática en la búsqueda del bien común.

V11: Indagación mediante métodos científicos para la construcción de sus conocimientos.

V12: Inversión por parte del Estado y/o colegio para la enseñanza de la EA

V13: Existencia de recursos y materiales para la enseñanza de la EA.

Figura 1. Motricidad y dependencia del sistema estudiado.

Fuente: elaboración propia (con apoyo del software Micmac). 
La matriz de motricidad y dependencia reconoce la llamada "zona de conflicto" (Godet, 2007), la cual comprende aquellas variables clave que "mueven" al sistema. Estas variables claves son:

a. Variable N.$^{\circ} 7$ (aprendizaje a partir de la realidad local a lo global): lo que significa que dentro del proceso de enseñanza-aprendizaje se debe contemplar el contexto y sus problemáticas locales para que el niño y la niña aprendan con miras a una perspectiva más global.

b. Variable N. ${ }^{\circ} 4$ (promoción de acciones sostenibles dentro y fuera del aula): donde el objetivo ideal de las instituciones educativas se basa en promover la gestión de recursos, el uso ecoeficiente del agua y energía, así como la búsqueda de un consumo sostenible a partir de la biodiversidad de su localidad.

c. Variable N. ${ }^{\circ} 3$ (acciones solidarias y de emprendimiento en la comunidad para el desarrollo sostenible): es decir, cuánto se toma en cuenta la interacción de los docentes con la comunidad para generar redes y alianzas estratégicas para fomentar y contribuir al desarrollo sostenible.

Las variables claves guardan relación estrecha con los actores sociales que las manejan. Estos tienen cierto grado de poder sobre las mismas. Se entiende el poder del actor como la capacidad de influir sobre otros dentro del sistema estudiado (Godet, 2007). Este grado de poder de los actores se estableció con ayuda de los mismos expertos, a través de un procedimiento similar al del análisis estructural. En una matriz relacional se calificó el grado de influencia de un actor sobre otro. Los actores sociales involucrados en el sistema de la EA para la niñez temprana peruana, en orden jerárquico de poder, fueron los siguientes:

1. Ministerio de Educación del Perú (Minedu).

2. Centros educativos para la niñez temprana.

3. Docentes de niñez temprana.

4. Padres y madres de familia (PP. MM. FF).
5. Ministerio del Ambiente del Perú (Minam).

6. Municipalidades distritales (regionales).

La primera variable clave se intercepta con las propuestas teóricas que menciona Novo (2006) sobre la relación de la naturaleza y el ser humano como "uno solo" y no como seres aislados. Constituyen una misma comunidad y un sentido de pertenencia del ser humano al mundo. La evidencia al obtener esta variable como gravitante para el sistema, coincide con Leff (1998), quien ya proponía orientar la acción pedagógica desde la realidad ecológica y cultural local para lograr aprendizajes significativos en los niños y las niñas. La segunda variable se sustenta en el hecho de que, como método pedagógico, se deben promover acciones sostenibles dentro y fuera del aula para satisfacer las necesidades del presente sin afectar a futuras generaciones (Sen, 2013; WCED, 1987). A partir de estas acciones se construye la identidad ambiental y se establece aquella moral requerida para un desarrollo sostenible (Gregers, 2013; Green, 2015; Eunhye et al., 2016). La tercera variable clave está vinculada a la importancia de la formación y competencias de los docentes. Esto coincide con lo ya reportado por McKeown (2007) y por la Unesco (2005). En este mismo sentido, la acción pedagógica compromete a los docentes como agentes de cambio para generar acciones sostenibles y redes con la comunidad local. Esto repercute directamente en los procesos de aprendizaje de los y las estudiantes (Bruns y Luque, 2014; Tan, 2015; Mizala y Schneider, 2019).

Por otro lado, resulta necesario mencionar la importancia que estas variables toman en contextos como los que vive el Perú y el mundo a raíz de la pandemia del covid-19. Por un lado, las repercusiones que las políticas de los países —en cuanto a salubridad y cuidado ambiental- pueden tener refuerzan esta primera idea de entender desde la niñez sobre los impactos globales (mundiales) de nuestros actos. En el caso peruano, los días de confinamiento obligatorio decretados por el Gobierno desde el mes de marzo del 2020 han 
repercutido de manera curiosa y favorable en acciones sostenibles de cuidado y limpieza del agua, de recuperación de sistemas biológicos y de consumo energético. Por ejemplo, el río Rímac, el más importante de la ciudad de Lima, ha quedado limpio y transparente, así como las playas aledañas al litoral limeño que han vuelto a ser visitadas por una biodiversidad de especies que no se veían desde tiempo atrás. Esto revalora la idea de formar desde temprano a nuestros niños en principios que favorezcan el cuidado y respeto de toda la fauna y flora que coexiste en nuestro entorno.

\section{Conclusiones}

El sistema de EA para la niñez temprana peruana posee tres variables claves que lo podrían determinar para el futuro en el contexto local: el aprendizaje a partir de la realidad local a lo global, la promoción de acciones sostenibles y las acciones solidarias y de emprendimiento de los docentes en la comunidad para el desarrollo sostenible. Su gestión y manejo recae especialmente en las acciones políticas que el Ministerio de Educación del Perú pueda desarrollar para el futuro.

La investigación advierte que las tres variables aludidas se encuentran en el cuadrante correspondiente a la zona de mayor motricidad y dependencia de la gráfica (zona de conflicto). En esta zona se ubican aquellas variables que terminan siendo claves por su alto nivel de sensibilidad para la estructura; ya que cualquier afectación o variación de las mismas implicaría un movimiento intensivo para todo el sistema analizado. Las tres variables mencionadas pertenecen a dos de las cuatro dimensiones inicialmente establecidas para del sistema. Esto permite reflexionar en cuanto a que el foco de atención política debe estar primordialmente orientada al docente, antes que a las competencias de los niños o los recursos de inversión por parte del Estado que, desde luego, no dejan de ser también fundamentales para la evolución del sistema. Concretamente, la vigilancia ha de centrarse en algunas competencias docentes en cuanto a su formación en EA, así como a reforzar ciertas metodologías empleadas en su proceso de enseñanza-aprendizaje. Este manejo de variables, desde luego, debe ser controlado escrupulosamente a partir de planes de acción que deben ser ejecutados a la luz de los escenarios futuros, cuya definición escapan al alcance de este trabajo.

En concordancia a lo anterior, algunas líneas de indagación que se abren a partir de esta evidencia se vinculan al hecho de pensar el desarrollo sostenible como un asunto ético y político, tal y como lo menciona Novo (2009). Por tanto, resulta un imperativo repensar las políticas educativas ambientales desde el Ministerio de Educación del Perú y desde el Ministerio del Ambiente (actor que no es predominante en el sistema de EA para la niñez peruana en la actualidad). Como señalan Greenall y Womersley (1977), las construcciones políticas que atiendan a la problemática ambiental aseguran el éxito a largo plazo. La identificación de estas variables clave en el sistema estudiado permite sentar las bases para crear un plan estratégico que direccione a las instituciones educativas y las prepare para atender futuros escenarios de la EA en la niñez temprana peruana.

\section{Referencias}

Adamson, P. (2008). The Child Care Transition: A league table of early childhood education and care in economically advanced countries. Innocenti Report Card, 8. Unicef. https://www. unicef-irc.org/publications/507-the-child-care-transition-a-league-table-of-early-childhood-education-and-care-in.html

Asian Development Bank (2015). Global Increase in Climate-Related Disasters. ADB Economics Working Paper Series, 466. https://www. adb.org/sites/default/files/publication/176899/ ewp-466.pdf

Barratt Hacking, E., Cutter-Mackenzie, A., Barratt, R. (2013). Children as active researchers. The potential of environmental education research involving children. En R. B. Stevenson, 
M. Brody, J. Dillon y A. E. J. Wals (eds.), International Handbook Of Research On Environmental Education (pp. 438-458). https://doi. org/10.4324/9780203813331.ch41

Bowers, C. (2002). Detrás de la apariencia: Hacia la descolonización de la educación. Bellido Ediciones SRL. http://www.pratec.org/wpress/ pdfs-pratec/detras-de-la-apariencia.pdf

Bruchner, P. (2012). Escuelas infantiles al aire libre. Cuadernos de Pedagogía, 420, 26-29.

Bruns, B., Luque, J. (2014). Great Teachers. How to Raise Student Learning in Latin America and the Caribbean. https://doi. org/10.1596/978-1-4648-0151-8

Carson, R. (1998). The sense of wonder. HarperCollins.

Castillo, A., Suárez, J., Mosquera, J. (2017). Naturaleza y sociedad: relaciones y tendencias desde un enfoque eurocéntrico. Revista Luna Azul, 44, 348-371. https://doi.org/10.17151/ luaz.2017.44.21

Clarke-Stewart, A., Allhusen, V. D. (2005). What We Know About Child Care. Harvard University Press.

Consejo Nacional de Educación (2020). Proyecto Educativo Nacional 2036. http://www.cne. gob.pe/uploads/publicaciones/2020/proyecto-educativo-nacional-al-2036.pdf

Davis, J. (1998). Young Children, Environmental Education, and the Future. Early Childhood Education Journal, 26(2), 117-123. https://doi. org/10.1023/A:1022911631454

De Jouvenel, B. (1964). Du principat. Revue française de science politique, 14(6), 1053-1086. https://doi.org/10.3406/rfsp.1964.403472

Díaz Flores, M. (2008). Reseña de "Diez nuevas competencias para enseñar" de Philippe Perrenoud. Tiempo de Educar, 9(17), 153-159.

Engdahl,I.(2015). EarlyChildhoodEducationforSustainability. The OMEP World Project. International Journal of Early Childhood, 47(3), 347-366. https://doi.org/10.1007/s13158-015-0149-6

Eunhye, P., Heejin, K., Sunyoung, Y. (2016). Perceptions and Attitudes of Early Childhood
Teachers in Korea About Education for Sustainable Development. International Journal of Early Childhood, 48(3), 369-385. https://doi. org/10.1007/s13158-016-0176-y

Fedosejeva, J., Boce, A., Romanova, M., Ilisko, D. (2018). Education for Sustainable Development. The Choice of Pedagogical Approaches and Methods for the Implementation of Pedagogical Tasks in the Anthropocene Age. Journal of Teacher Education for Sustainability, 20(1), 157179. https://doi.org/10.2478/jtes-2018-0010

Fjørtoft, I. (2001). The Natural Environment as a Playground for Children: The Impact of Outdoor Play Activities in Pre-Primary School Children. Early Childhood Education Journal, 29(2), 111-117. https://doi. org/10.1023/A:1012576913074

Flórez-Yepes, G., Rincón-Santamaría, A., Cardona, P., Gallego, F. (2018). Herramientas de aprendizaje para favorecer la educación ambiental. Caso de estudio Fundación Niños de Los Andes sede Manizales, Colombia. Revista Electrónica Educare, 22(2), 1-21. https://doi. org/10.15359/ree.22-2.5

Fondo de las Naciones Unidas para la Infancia (2010). Humanitarian action. Partnering for children in emergencies. https://static.unicef. org/har2010/files/UNICEF Humanitarian Action Report 2010-Full Report WEB EN.pdf

Freire, H. (2011). Educar en verde. Graó.

Fundación Universitaria Iberoamericana (2020). Educación Ambiental. https://www.funiber. org/educacion-ambiental\#field-objetivos

Galeano, J., Parra, C., Chocontá, J. (2018). Educación ambiental en la primera infancia: Una mirada en Latinoamérica. https://ebookcentral.proquest.com/lib/universidadcomplutense-ebooks/detail.action?docID $=5759287$

Giollito, P. (1984). Pedagogía del Medio Ambiente. Herder.

Godet, M. (2007). Prospectiva Estratégica: Problemas y métodos. http://www. laprospective.fr/dyn/francais/memoire/Cajadeherramientas2007.pdf 
Gouvêa, G. (2006). Rumos da formação de professores para a Educação Ambiental. Educar em revista, 27, 163-179. https://doi.org/10.1590/ $\underline{\text { S0104-40602006000100011 }}$

Green, C. J. (2015). Toward Young Children as Active Researchers. A Critical Review of the Methodologies and Methods in Early Childhood Environmental Education. The Journal of Environmental Education, 46(4), 207-229. https://doi.org/10.1080/00958964.2015.105 $\underline{0345}$

Greenall, A., Womersley, J. (1977). Development of Environmental Education in Australia-Key Issues. Curriculum Development Centre.

Gregers, K. (2013). Why Education for Sustainable Development needs Early Childhood Education. The case of Norway. Journal of Teacher Education for Sustainability, 15(1), 107-120. https://doi.org/10.2478/jtes-2013-0007

Instituto Nacional de Estadística e Informática (2017). Características de la población. https:// www.inei.gob.pe/media/MenuRecursivo/publicaciones digitales/Est/Lib1539/cap01.pdf

Intergovernmental Panel on Climate Change (2013). Climate change 2013: The physical science basis. Contribution of working group I to the fifth assessment report of the intergovernmental panel on climate change, 1535. http://www.climatechange2013.org/images/ report/WG1AR5 Frontmatter FINAL.pdf

International Union for Conservation of Nature (1972). Environmental Education in an Urban Society. https://portals.iucn.org/library/sites/library/files/documents/NS-SP-037.pdf

La prospective (octubre de 2019). MicMac. Métodos de prospectiva. Micmac. http://es.laprospective.fr/Metodos-de-prospectiva/Los-programas/67-Micmac.html

Leff, E. (1998). Saber ambiental. ustentabilidad, racionalidad, complejidad, poder. https:// bibliodarq.files.wordpress.com/2014/12/ leff-e-saber-ambiental-sustentabilidad-racionalidad-complejidad-poder.pdf
Louv, R. (2008). The last child in the woods. Algonquin. https://doi.org/10.12968/ sece.2008.2.1086

Lowe, D., Pierce, K., Burchinal, M. (2016). Early child care and adolescent functioning at the end of high school. Results from the NICHD Study of Early Child Care and Youth Development. Developmental Psychology, 52(10), 1634-1645. https://doi.org/10.1037/dev0000169

Marcén, C. (2014). Biodiversidad a la baja, educación al rescate. Cuadernos de Pedagogía, 446, 74-78.

McKeown, R. (2007). Good practices in education for sustainable development. Teacher education institution (documento de programa o de reunión n. ${ }^{\circ}$ 1). https://unesdoc.unesco.org/ ark:/48223/pf0000152452

Ministerio de Educación del Perú (2016). Curriculo Nacional de la Educación Básica. http://www.minedu.gob.pe/curriculo/

Ministerio de Educación del Perú (2019a). Educación Ambiental. Minedu. http://www.minedu. gob.pe/educacion-ambiental/

Ministerio de Educación del Perú (2019b). Marco normativo. Línea de tiempo. Enfoque Ambiental Minedu. http://www.minedu.gob.pe/ educacion-ambiental/

Ministerio de Educación del Perú (2019c). Política nacional de educación ambiental 20162021 (PNEA). http://www.minedu.gob.pe/ educacion-ambiental/

Ministerio del Ambiente, Ministerio de Educación del Perú (2016). Plan Nacional de Educación Ambiental 2017-2022. http://www.minam. gob.pe/wp-content/uploads/2016/12/plan-nacional-educacion-ambiental-2017-2022.pdf

Mishra, S. (2016). Environmental Education. Principles and Policies. Kalpaz Publications.

Mizala, A., Schneider, B. (2019). Promoting quality education in Chile. The politics of reforming teacher careers. Revista de Política Educativa, 1-27.

Montero, M. (2009). Emilio. Niño y Educación. Magistro, 1(5), 59-73. https://doi.org/10.15332/ $\underline{\mathrm{s} 2011-8643.2009 .0005 .03}$ 
Morachimo, L., Piscoya, L. (2004). Temas transversales y desarrollo sostenible. Claves para su inserción en la institución educativa. CIFO.

Munck, R., McConnell, G. (2009). University strategic planning and the foresight/futures approach. Planning for Higher education, 38, 31-40.

NICHD Early Child Care Research Network (2005). Child Care and Children's Development. Results from the NICHD Study of Early Child Care and Youth Development. Guilford Press.

Novo, M. (2006). El desarrollo sostenible. Su dimensión ambiental y educativo. Unesco; Pearson.

Novo, M. (2009). La educación ambiental, una genuina educación para el desarrollo sostenible. Revista de Educación, 195-217.

Novo, M., Murga, M. Á. (2010). Educación ambiental y ciudadanía planetaria. Revista Eureka Sobre Enseñanza y Divulgación de Las Ciencias, 7, 179-186. https://doi.org/10.25267/Rev Eureka ensen divulg_cienc.2010.v7.iextra.03

Organización de las Naciones Unidas (2006). Observación general $n .{ }^{\circ} 7$ (2005). Implementación de los derechos del niño en la primera infancia. https://www.ohchr.org/ layouts/15/WopiFrame.aspx?sourcedoc=/Documents/Issues/Women/WRGS/Health/GC7. pdf\&action $=$ default\&DefaultitemOpen $=1$

Organización de las Naciones Unidas (2019). Objetivos de desarrollo sostenible. https://www.un.org/sustainabledevelopment/ es/objetivos-de-desarrollo-sostenible/

Organización de las Naciones Unidas (2020). La agenda para el desarrollo sostenible. https://www.un.org/sustainabledevelopment/ es/development-agenda/

Organización de las Naciones Unidas para la Educación, la Ciencia y la Cultura (2005). Directrices y recomendaciones encaminadas a reorientar la formación de docentes para abordar el tema de la sostenibilidad (documento de programa o de reunión n. ${ }^{\circ}$ 2). https://unesdoc. unesco.org/ark:/48223/pf0000143370_spa
Ruiz-Ruiz, M. F. (2017). La prospectiva francesa como estrategia de planeación universitaria: evaluación de la aceptabilidad de su aplicación en una facultad de ingeniería (tesis de doctorado). Pontificia Universidad Católica del Perú. Repositorio institucional de la Pontificia Universidad Católica del Perú. http://tesis.pucp.edu. pe/repositorio/handle/20.500.12404/7929

Russo, S. (2001). Promoting attitudes toward E.E. depends on early childhood education: What view do we hold? Australian Science Teachers' Association, 17(4), 34-36.

Sen, A. (2013). The Ends and Means of Sustainability. Journal of Human Development and Capabilities, 14(1), 6-20. https://doi.org/10.1080 /19452829.2012.747492

Sistema Nacional de Información Ambiental (2020). Superficie de área verde urbana por habitante. https://sinia.minam.gob.pe/indicadores/ superficie-area-verde-urbana-habitante

Tan, O. S. (2015). Teacher Polices. Global Best Practices for Developing the Teaching Profession (World Innovation Summit for Education WISE). https://www.wise-qatar.org/sites/default/files/asset/document/wise-research-2-nie-11 17.pdf

Tanner, T. (2010). Shifting the narrative: Child-led responses to climate change and disasters in El Salvador and the Philippines. Philippines. Children \& Society, 24(4), 339-351. https://doi. org/10.1111/j.1099-0860.2010.00316.x

Tonucci, F. (2019). La ciudad de los niños: ¿por qué necesitamosdelosniñosparasalvarlasciudades? https://www.gob.mx/cms/uploads/attachment/ file/477029/FCN TEXTO TONUCCI.pdf

Tonucci, F. (2020). City of Children. Vernon Press. Torquati, J., Cutler, K., Gilkerson, D., Sarver, S. (2013). Early Childhood Educators' Perceptions of Nature, Science, and Environmental Education. Early Education and Development, 24(5), 721-743. https://doi.org/10.1080/1040 $\underline{9289.2012 .725383}$

Torrico, G. (2020). El espacio que compartimos. https://f.rpp-noticias.io/2020/02/26/infor$\underline{\text { me-el-espacio-que-compartimos 906000.pdf }}$ 
United States Green Building Council (2018). Green Classroom Professional Certificate. $\quad$ https://www.usgbc.org/education/ sessions/green-classroom-professional-certificate-11781677\#

US Global Change Research Program (2018). Fourth National Climate Assessment. Impacts, Risks, and Adaptation in the United States. https://nca2018.globalchange.gov/downloads/ NCA4 2018 FullReport.pdf

Waller, T., Sandseter, E., Wyver, S., Ärlemalm-Hagsér, E., Maynard, T. (2010). The dynamics of early childhood spaces: opportunities for outdoor play? European Early Childhood
Education Research Journal, 18, 437-443. https://doi.org/10.1080/1350293X.2010.525917 Wensing, E., Torre, C. (2009). The Ecology of Education. Knowledge Systems for Sustainable Development and Sustainability. Journal of Teacher Education for Sustainability, 11(1), 3-17. https://doi.org/10.2478/ v10099-009-0028-z

World Commission on Environment and Development (1987). Our common future. Report of the World Commission on Environment and Development. https://sustainabledevelopment. un.org/content/documents/5987our-common-future.pdf 